\title{
Relação entre obesidade, insulina plasmática e posicionamento da falange distal em equinos da raça Crioula
}

\author{
[Relationship between obesity, insulin and placement of plasma in equine \\ distal phalanx of breed creole] \\ C.F.R. Paz', J.C. Paganela ${ }^{2}$, C.A. dos Santos $^{3}$, C.E.W. Nogueira ${ }^{4}$, R.R. Faleiros ${ }^{5}$ \\ ${ }^{1}$ Mestrando em Ciência Animal - Universidade Federal de Minas Gerais - Belo Horizonte, MG \\ ${ }^{2}$ Residente - Clínica Horse Center - Pedro do Rio, RJ \\ ${ }^{3}$ Médico veterinário autônomo - Pelotas, RS \\ ${ }^{4}$ Faculdade Veterinária - Universidade Federal de Pelotas - Pelotas, RS \\ ${ }^{5}$ Escola de Veterinária - Universidade Federal de Minas Gerais - Belo Horizonte, MG
}

\begin{abstract}
RESUMO
Verificaram-se características radiográficas do posicionamento da falange distal em relação ao estojo córneo em equinos da raça Crioula e correlacionaram-se tais achados com sinais de obesidade e concentrações plasmáticas de insulina. Consideraram-se dois grupos com base no escore da condição corporal (ECC). A média de idade entre os grupos foi de 9,2 $\pm 5,8$ anos em indivíduos normais (ECC 5-7) e de 10,3 $\pm 3,7$ anos em obesos (ECC 8-9). Diferenças estatísticas entre grupos ocorreram para valores de peso, perímetro torácico, escore da condição corporal, escore da crista do pescoço, comprimento do pescoço, circunferência do pescoço em três regiões referentes a $25 \%, 50 \%$ e $75 \%$ do comprimento do pescoço e entre o posicionamento da falange distal em relação ao estojo córneo. O ângulo formado entre as superfícies dorsais de falange distal e casco (ângulo de rotação) correlacionou-se estatisticamente com ECC $(r=0,30 ; \mathrm{P}=0,02)$ e com peso e perímetro torácico $(r=0,50 ; P<0,01)$. Quanto à concentração de insulina plasmática, foram encontradas correlações positivas com ECP $(r=0,40 ; \mathrm{P}<0,01)$ e com ângulo de rotação $(r=0,23 ; P=0,08)$, e correlação negativa com idade $(r=-0,42 ; P<0,01)$. Em oito animais (27\%) o ângulo de rotação foi maior que 2 graus; destes, $25 \%$ eram normais e $75 \%$, obesos $(P<0,05)$. Aparentemente, em equinos da raça Crioula, características morfométricas no casco diferiram dos padrões internacionais obtidos de outras raças. A obesidade interferiu na relação espacial da falange distal com o estojo córneo, indicando que os animais obesos dessa raça são mais propensos a desenvolver laminite.
\end{abstract}

Palavras-chave: equino, raça Crioula, obesidade, insulina, laminite

\begin{abstract}
The radiographic relationship of the distal phalanx with the hoof capsule was verified in Creole breed horses and these findings correlated with signs of obesity and insulin blood levels. Horses were divided in two groups based on their body score condition (ECC). The average age was 9.2 \pm 5.8 years in normal (ECC 5-7) and $10.3 \pm 3.7$ years in the obese group (ECC 8-9). Statistical differences between groups $(P<0.05)$ were detected for values of weight, thoracic girth, body score condition, neck score (ECP), neck length, neck circumference into three regions based on 25\% (P25), 50\% (P50) and 75\% (P75) of the length of the neck and phalanx and hoof capsule relationship. The angle between the dorsal aspects of distal phalanx and hoof wall (rotation angle) statistically correlated with ECC $(r=0.30 ; P=0.02)$ and with weight and thoracic girth $(r=0.50$; $P<0.01)$. Plasma insulin concentrations positively correlated with ECP $(r=0.40, P<0.01)$ and rotation angle $(r=0.23, P=0.08)$ and negatively with age $(r=-0.42, P<.01)$. In eight animals $(27 \%)$ rotation angle was greater than 2 degrees, of which $25 \%$ were normal and obese $75 \%(P<0.05)$. Apparently in Creole horses, morphometric characteristics of the hoof differ from international standards obtained from other breeds. Obesity interfered with the spatial relationship of the distal phalanx with the hoof capsule, indicating that obese animals of this breed are more likely to develop laminitis.
\end{abstract}

Keywords: equine, Creole breed, obesity, insulin, laminitis

Recebido em 9 de junho de 2012

Aceito em 30 de julho de 2013

*Autor para correspondência (corresponding author)

E-mail: faleirosufmg@gmail.com 


\section{INTRODUÇÃO}

Distúrbios endócrinos associados à obesidade têm sido relatados como uma das principais causas de laminite em equinos em todo o mundo. Recentemente, o termo síndrome metabólica equina (SME) foi aceito pela Associação Americana de Medicina Interna (Frank et al., 2010), para descrever o aumento da adiposidade associada à resistência à insulina que caracteriza as alterações endócrinas. A SME é relatada em diversas raças de pôneis e em cavalos das raças Morgan, Paso Fino, Árabe, Quarto de Milha, Saddlebreds e Tennessee Walking (Frank, 2011), e está relacionada com o padrão nutricional contemporâneo e com a falta de atividade física adequada, a que a espécie equina é submetida.

A adiposidade regional em cavalos e pôneis, em especial o tecido adiposo depositado ao longo da crista do pescoço, tem sido relacionada com problemas metabólicos (Johnson, 2002; Treiber et al., 2006; Carter et al., 2009). Esse tecido libera normalmente pequenas quantidades de citocinas, também chamadas de adipocinas, que exercem funções autócrinas e parácrinas. No entanto, em indivíduos obesos, altas concentrações dessas citocinas são excretadas para além dos tecidos de origem. Em elevada quantidade na circulação sanguínea, moléculas pró-inflamatórias atuam nos receptores de insulina, contribuindo com o desenvolvimento da resistência à insulina (RI), segundo Johnson et al. (2010).

Os efeitos maléficos da obesidade sobre a saúde dos equinos ainda não estão bem definidos como em humanos. Contudo, a associação entre obesidade e laminite está bem delimitada e já alcança níveis alarmantes. Por exemplo, estudos recentes realizados na Finlândia demonstraram que, em sua grande maioria, os casos de laminite apresentados para hospitalização estavam associados à endocrinopatia, sendo $95 \%$ dos equinos com laminite obesos e $89 \%$ deles diagnosticados com hiperinsulinemia (Karikoski et al., 2011).

A laminite associada à endocrinopatia tem características distintas da laminite subsequente às alterações sépticas. Seu curso é subclínico, e importantes alterações na estrutura do casco podem ocorrer, mesmo antes da presença dos primeiros sinais clínicos. Dessa forma, o diagnóstico precoce é a principal forma de controle desse tipo de laminite (Walsh, 2009).

Os cavalos Crioulos, em sua origem, passaram fome, sede, foram perseguidos por homens e predadores e precisaram suportar temperaturas extremas, desde as fortes geadas do inverno até o rigoroso sol do verão (Coelho, 2012). Esse processo naturalmente selecionou indivíduos mais resistentes e com melhor conversão alimentar, que formaram a base genética da raça. Hoje, o Crioulo está entre as raças brasileiras mais valorizadas. Em busca de precocidade e do acúmulo de depósitos de gordura que proporcionam uma aparência externa em formas arredondadas - de grande valor para alguns criadores -, os cavalos têm sido alimentados com alimentos de alto valor nutricional. Assim, a obesidade tem se tornado evidente nessa raça e, dessa forma, possíveis distúrbios endócrinos tornam-se motivo de preocupação para veterinários e proprietários, principalmente quanto às alterações subclínicas relacionadas à laminite.

O presente estudo é um dos trabalhos que estão sendo desenvolvidos em relação à obesidade em raças brasileiras de cavalos. A hipótese é que equinos obesos de raças nacionais são propensos à hiperinsulinemia e à laminite e que alterações na relação espacial entre a falange distal e o estojo córneo podem ocorrer nesses animais mesmo antes que qualquer alteração clínica sugestiva de laminite seja detectada. O objetivo foi verificar a incidência das alterações radiográficas do posicionamento da falange distal em relação ao estojo córneo, em equinos da raça Crioula, e correlacionar tais achados com a presença de obesidade e hiperinsulinemia.

\section{MATERIAL E MÉTODOS}

O experimento foi aprovado pelo Conselho de Ética e Experimentação Animal da Universidade Federal de Pelotas. Avaliaram-se 30 éguas da raça Crioula, com idades entre 3 e 19 anos, sendo oriundas de dois criatórios de cavalos Crioulos, localizados em uma região de clima temperado, na cidade de Pelotas ( $31^{\circ} 46^{\prime} 19^{\prime \prime}$ S $52^{\circ} 20^{\prime} 34^{\prime}$ ' O), no Rio Grande do Sul. Os animais não apresentavam histórico de claudicação e tampouco sinais clínicos de laminite. As avaliações foram realizadas no período de novembro de 2010 a janeiro de 2011. As éguas, 
criadas em sistema semi-intensivo, eram mantidas em pastagem cultivada de azevém (Lolium multiflorum) no inverno e milheto (Pennisetum americanum) no verão, e recebiam $1 \%$ do PV/dia de suplementação que continha $12 \%$ PB e $3.000 \mathrm{Mcal} / \mathrm{kg}$, em duas porções durante o dia, em intervalo de 12 horas.

Com o intuito de verificar e classificar a obesidade e a adiposidade, os animais foram pesados e mensurados com uma fita métrica, para obtenção do perímetro torácico (PT). Foram avaliados quanto ao escore de condição corporal (ECC), segundo Hennek et al. (1983), em uma escala de 1 a 9. Com essa escala determinaramse dois grupos: com escore de 5 a 7 - indivíduos normais -, e escore 8 e 9 - obesos.

A fim de avaliar especificamente a adiposidade na região do pescoço, utilizou-se o método descrito por Carter et al. (2009), que estabelece escore de 0 a 5 para a espessura da crista do pescoço (ECP). Mais além, foram realizadas mensurações de pescoço, segundo Frank et al. (2006). O comprimento (CP) foi medido, com a cabeça posicionada em um ângulo de $45^{\circ}$, do topo até o ponto mais alto da cernelha. A circunferência do pescoço foi, então, mensurada a partir de três pontos dorsais distintos, correspondentes a 25\% (P 25), 50\% (P 50) e 75\% (P 75) do comprimento do pescoço.

Como índice de obesidade, o valor de P 50 (crista do pescoço) foi dividido pela medida de altura da cernelha (h2), segundo determinação de Carter et al. (2009). Para investigar a possível incidência de hiperinsulinemia, amostras de $10 \mathrm{~mL}$ de sangue foram obtidas da veia jugular esquerda, com o uso de tubos que continham EDTA do tipo Vacutainer®, antes do fornecimento da ração da manhã, ou seja, pelo menos 12 horas depois da última refeição com concentrado. $\mathrm{O}$ sangue foi imediatamente resfriado e mantido em isopor, com gelo, por período máximo de duas horas pós-coleta. Os tubos foram centrifugados por 30min a 1.200rpm, para a separação do plasma. Alíquotas de $1 \mathrm{~mL}$ de plasma foram coletadas e armazenadas a $-20^{\circ} \mathrm{C}$, durante cinco meses, até serem realizadas as análises. Para mensurar a concentração de insulina plasmática, as análises foram realizadas pelo BET Laboratories ${ }^{\mathrm{TM}}$ Brasil, e os valores entre $2-25 \mu \mathrm{UI} / \mathrm{mL}$ foram considerados como fisiológicos.
Com o propósito de verificar possíveis presenças de claudicação, sensibilidade dolorosa do casco na superfície solear e presença de pulso digital palmar exarcebado que pudessem justificar possíveis alterações radiográficas, foi realizada avaliação do sistema locomotor, de acordo com Stashak (2002), e radiografadas apenas as éguas que não apresentaram alterações. Exposições radiográficas dos cascos dos membros torácicos foram realizadas em projeção látero-medial de forma padronizada, utilizando um aparelho de raio-x portátil modelo MinxRay TR90 Tough Ray e regime de $64 \mathrm{kV}$ e 0,04 de tempo de exposição.

Após revelação, as imagens foram digitalizadas e avaliadas. Mensuraram-se individualmente para cada casco: o ângulo da superfície dorsal da parede do casco em relação ao solo (AC) e o ângulo entre a superfície dorsal da falange distal em relação ao solo (AF). O ângulo formado entre as superfícies dorsais de falange distal e casco (ângulo de rotação) foi obtido subtraindo-se AC de AF. Para efeito de comparação entre os grupos, consideraram-se como anormais valores de ângulo de rotação, iguais ou acima de 2 graus positivos. Esse valor foi estabelecido com base em experimento prévio realizado na raça Mangalarga Marchador, em que o valor médio nos animais normais - escore corporal entre 5 e 8 - foi de $-2,18^{\circ}$ (Lima et al., 2011). Também foi determinada, em porcentagem, a proporção (PP) da distância entre as superfícies dorsais da falange distal e do casco em relação à distância entre a ponta da falange distal até sua superfície articular com o sesamóide distal, conforme proposto por Pollitt (2008).

Para comparação entre grupos, utilizou-se o teste t para os dados paramétricos e o teste MannWhitney para dados não paramétricos. Também estimaram-se as correlações entre idade, achados de características corporais, concentração de insulina e medidas radiográficas pelos testes de Pearson para dados paramétricos e Spearmann para não paramétricos. A associação entre o posicionamento da falange distal em relação ao ângulo de rotação e o escore corporal foi estimada por meio do teste exato de Fisher. Para todos os testes, considerou-se $\mathrm{P} \leq 0,05$ como significativo e $\mathrm{P} \geq 0,06$ a $\mathrm{P}<0,10$ com tendência a ser significativo. 


\section{RESULTADOS}

Os dados referentes à idade e características corporais dos dois grupos estão apresentados na Tabela 1. Diferenças significativas entre grupos não foram encontradas apenas para idade $\mathrm{e}$ comprimento de pescoço.

Os dados referentes ao exame radiográfico e à concentração plasmática de insulina plasmática estão na Tabela 2. A média ( $\pm \mathrm{DP})$ geral para PP foi de $34 \pm 4,5 \%$. Observou-se apenas uma tendência $(\mathrm{P}=0,09)$ de os valores do grupo obeso serem ligeiramente mais altos que os do grupo normal $-35,4 \pm 3,5$ e $33 \pm 4,5$, respectivamente. A concentração média geral para insulina plasmática foi de $2,44 \pm 1,99 \mu \mathrm{UI} / \mathrm{mL}$, não havendo diferença entre grupos.

Observaram-se correlações significativas entre diferença de ângulos com peso corporal e perímetro torácico $(\mathrm{r}=0,50 ; \mathrm{P}<0,01)$ e com escore corporal $(\mathrm{r}=0,30 ; \mathrm{P}=0,02)$. A concentração de insulina plasmática correlacionou-se positivamente com escore corporal e com escore de pescoço $(r=0,40$; $\mathrm{P}<0,01)$ e negativamente com idade ( $\mathrm{r}=-0,42$; $\mathrm{P}<0,01)$, e observou-se correlação entre concentração de insulina e rotação de falange ( $\mathrm{r}$ $=0,23 ; \mathrm{P}=0,08)$. Associação significativa foi observada entre obesidade e ângulo de rotação de falange. De todas as éguas, $27 \%$ tinham ângulo de rotação maior que 2 graus. Destas, $25 \%$ eram normais e $75 \%$ obesas $(\mathrm{P}<0,05)$ (Tab. 3). Em todos os animais a alteração foi unilateral.

$\mathrm{Na}$ associação entre ângulo de rotação de falange e concentração plasmática de insulina $(\mathrm{P}<0,08)$, as duas éguas com maiores valores de insulina plasmática, mesmo estando em grupos distintos, apresentaram sinais de rotação de falange, isto é, DIF ANG maior que 2 graus.

Tabela 1. Média e desvio padrão (DP) de idade e características corporais de éguas Crioulas normais e obesas

\begin{tabular}{|c|c|c|c|c|c|}
\hline \multirow[t]{2}{*}{ Variável } & \multicolumn{2}{|c|}{ Normal } & \multicolumn{2}{|c|}{ Obesa } & \multirow[b]{2}{*}{ Valor de P* } \\
\hline & Média & DP & Média & DP & \\
\hline Idade & 9,2 & $\pm 5,7$ & 10,33 & $\pm 3,7$ & $\mathrm{P}=0,4084$ \\
\hline Peso (kg) & 449,4 & $\pm 39,1$ & 523,2 & $\pm 31,7$ & $\mathrm{P}<0,0001$ \\
\hline PT $(\mathrm{cm})$ & 179,8 & $\pm 6,3$ & 190,7 & $\pm 4,0$ & $\mathrm{P}<0,0001$ \\
\hline $\mathrm{ECC}$ & 6,5 & $\pm 0,6$ & 8,2 & $\pm 0,4$ & $P<0,0001$ \\
\hline ECP & 2,7 & $\pm 0,9$ & 3,3 & $\pm 0,9$ & $\mathrm{P}=0,0202$ \\
\hline $\mathrm{CP}(\mathrm{cm})$ & 76,8 & $\pm 5,7$ & 79,25 & $\pm 7,0$ & $\mathrm{P}=0,1365$ \\
\hline P $25(\mathrm{~cm})$ & 75,7 & $\pm 3,3$ & 81,25 & $\pm 5,0$ & $\mathrm{P}<0,0001$ \\
\hline P $50(\mathrm{~cm})$ & 91,6 & $\pm 5,2$ & 98,50 & $\pm 7,2$ & $\mathrm{P}<0,0001$ \\
\hline P $75(\mathrm{~cm})$ & 105,8 & $\pm 5,8$ & 112,7 & $\pm 7,7$ & $\mathrm{P}=0,0002$ \\
\hline P 50/ h2 & 66,07 & $\pm 3,7$ & 69,27 & $\pm 4,2$ & $\mathrm{P}=0,0075$ \\
\hline
\end{tabular}

*Teste t para variáveis paramétricas e Mann-Whitney para não paramétricas $(\mathrm{P} \leq 0,05)$.

PT: perímetro torácico; ECC: escore de condição corporal; ECP: escore de gordura da crista do Pescoço; CP: comprimento de pescoço; P 25: circunferência no ponto referente a 25\% do comprimento do pescoço; P 50: circunferência no ponto referente a $50 \%$ do comprimento do pescoço; P 75: circunferência no ponto referente a $75 \%$ do comprimento do pescoço; P 50/h2: circunferência do P 50 dividido pela altura da cernelha.

Tabela 2. Média e desvio padrão (DP) das variáveis radiográficas e concentração plasmática de insulina em éguas Crioulas normais e obesas

\begin{tabular}{|c|c|c|c|c|c|}
\hline \multirow{2}{*}{ Variável } & \multicolumn{2}{|c|}{ Normal } & \multicolumn{2}{|c|}{ Obesa } & \multirow{2}{*}{ Valor de $\mathrm{P}^{*}$} \\
\hline & Média & DP & Média & DP & \\
\hline Insulina $(\mu \mathrm{UI} / \mathrm{mL})$ & 2,36 & $\pm 2,27$ & 2,55 & $\pm 1,5$ & $\mathrm{P}=0,7276$ \\
\hline Ângulo de rotação $\left(^{\circ}\right)$ & $-1,6$ & $\pm 2,6$ & 0,22 & $\pm 2,18$ & $\mathrm{P}=0,0086$ \\
\hline $\mathrm{PP}(\%)$ & 33,3 & $\pm 4,5$ & 35,4 & $\pm 3,5$ & $\mathrm{P}=0,0895$ \\
\hline
\end{tabular}

*Teste t para variáveis paramétricas $(\mathrm{P} \leq 0,05)$.

DIF ANG: subtração do ângulo entre a superfície dorsal da parede do casco em relação ao solo (AC) e o ângulo entre a superfície dorsal da falange distal em relação ao solo (AF); PP: proporção da distância entre as superfícies dorsais da falange distal e do casco em relação à distância entre a ponta da falange distal até sua superfície articular com o sesamóide distal. 
Tabela 3. Número de éguas da raça Crioula normais e obesas com DIF ANG maior ou menor que 2 graus

\begin{tabular}{lcc}
$\begin{array}{l}\text { Ângulo de } \\
\text { rotação }\end{array}$ & Normal & Obesa \\
\hline$>2^{\circ}$ & 2 & 6 \\
$<2^{\circ}$ & 16 & 6 \\
Total & 18 & 12 \\
\hline p<0.05. & \\
$\begin{array}{l}\text { DIF ANG: subtração do ângulo entre a superfície } \\
\text { dorsal da parede do casco em relação ao solo (AC) e o }\end{array}$ \\
$\begin{array}{l}\text { ângulo entre a superfície dorsal da falange distal em } \\
\text { relação ao solo (AF). }\end{array}$
\end{tabular}

\section{DISCUSSÃO}

Não houve diferença em relação à idade nem ao comprimento do pescoço entre grupos, mas todas as outras características, indicadoras de adiposidade, apresentaram valores mais altos no grupo de éguas obesas. Enquanto a pontuação da condição corporal é um método aceitável para a avaliação da adiposidade global, a pontuação da crista do pescoço seria uma alternativa objetiva para os sistemas de pontuação subjetivos e também para padronizar a avaliação da distribuição regional de gordura na crista do pescoço (Carter et al., 2009). De acordo com o esperado, éguas Crioulas obesas apresentaram maior escore de crista de pescoço e maior diâmetro de pescoço que os de éguas controle.

No presente estudo, o grupo de éguas obesas apresentou média 3,3 para ECP. Para Walsh et al. (2009), a pontuação para adiposidade da crista do pescoço foi acima de 3 em todos os cavalos com SME e em metade dos cavalos com doença de Cushing. Esse valor também serve como um indicador fenotípico, que está associado com resistência à insulina e tendência para desenvolver laminite (Treiber et al., 2005; Carter et al., 2009; Walsh et al., 2009).

De forma inesperada, nenhum dos animais estudados apresentou aumento na concentração plasmática de insulina matinal. Estudos têm revelado que a deposição de gordura no pescoço é um dos principais indicadores de hiperinsulinemia no cavalo (Carter et al., 2009). Cavalos com pescoço "cristado" (cresty neck) têm $18,9 \%$ mais chances de apresentarem hiperinsulinemia (Carter et al., 2009). Mais além, os valores encontrados para insulina sérica basal foram mais baixos que os relatados em éguas Puro Sangue Inglês (PSI) sem obesidade ou qualquer sinal de claudicação (De Laat et al., 2012).

Alguns fatores poderiam estar relacionados à baixa concentração plasmática de insulina. Ou esses seriam valores fisiológicos, em virtude de não existirem relatos desses parâmetros na raça Crioula, ou, ainda, poderia ter havido redução na concentração de insulina devido ao período de tempo que as amostras levaram para serem processadas. Também há de se considerar que o método aqui utilizado de uma única avaliação de insulina plasmática não é a forma mais eficaz de identificar equinos com resistência insulínica. Os mais indicados são os testes dinâmicos, que envolvem a administração prévia de glicose combinada ou não com insulina, seguida da mensuração serial de insulina no sangue (Johnson et al., 2010).

As correlações significativas observadas entre teor de insulina e escore corporal e de crista de pescoço - além do fato de as duas éguas com maior concentração plasmática de insulina serem justamente as que apresentavam maior grau de rotação de falange -, podem ser consideradas como indicativas de uma associação entre insulina e laminite também na raça Crioula.

Os resultados da análise radiográfica foram consistentes ao demonstrarem, por meio da comparação entre grupos, pelos testes de correlação e pelo teste de Fisher, que a obesidade pode ser um fator determinante na relação espacial entre estojo córneo e terceira falange em equinos Crioulos. Até onde se sabe, este é o primeiro estudo controlado a demonstrar que equinos obesos estão mais predispostos a apresentar alterações no posicionamento da falange distal sem apresentar nenhum histórico ou sinal clínico de laminite. Tais achados demonstram que a avaliação radiográfica do casco de equinos obesos pode ser de grande valia no diagnóstico precoce de problemas metabólicos e laminite em equinos.

Os achados do presente estudo corroboram observações clínicas prévias de que esses casos de laminite são resultado de uma enfermidade crônica e subclínica de longa duração, em que o deslocamento da falange distal precede a 
sintomatologia dolorosa (Walsh, 2009). Estudo recente demonstrou que a infusão contínua de glicose para induzir hiperglicemia resultou na ocorrência de alterações histológicas importantes no casco em equinos PSI, sem que nenhum dos animais apresentasse claudicação (De Laat et al., 2012).

A alteração espacial da falange distal em relação ao estojo córneo aqui registrada em éguas Crioulas é um processo ainda não totalmente elucidado. Sabe-se que as células epidermais do tecido laminar são extremamente dependentes de glicose (Pass et al., 1998) e, consequentemente, poderiam ser afetadas pela resistência insulínica característica da obesidade. Contudo, foi relatado que a captação de glicose nas lâminas do casco não parece ser dependente de insulina (Asplin et al., 2007; Walsh et al., 2009). No entanto, esse hormônio tem ações vasorreguladoras, que representam uma ligação plausível entre RI e laminite em equinos (Frank et al., 2010). Recentemente foi relatado que neutrófilos oriundos de equinos obesos exibem capacidade oxidativa maior que os isolados de equinos normais (Holbrook et al., 2012). Esse fator pode ser importante, uma vez que infiltração leucocítica tem sido consistentemente detectada na fase de desenvolvimento de laminite em todos os modelos até então utilizados, ou seja, extrato de nogueira preta (Black et al., 2006, Faleiros et al., 2009), excesso de amido (Faleiros et al., 2011), oligofructose (Visser e Pollitt, 2011) e hiperinsulinemia (De Laat et al., 2012).

Um achado radiográfico de interesse foi sobre a relação da distância entre as superfícies dorsais da parede do casco e da falange distal em relação à distância da ponta da falange à superfície articular desta com o navicular (PP). Entre os grupos de indivíduos normais e obesos, observaram-se valores de $33,3 \%$ e $35,4 \%$, respectivamente (Tab. 2). Esses resultados são mais altos que os citados na literatura, que atribui um máximo de aproximadamente $25 \%$ para essa proporção em cavalos normais (Pollitt, 2008). Por se tratar de um dado obtido de cavalos hígidos, este achado indica um possível padrão de normalidade diferenciado da raça Crioula em relação às raças internacionais.

\section{CONCLUSÕES}

Equinos da raça Crioula possuem características morfométricas no casco que diferem de padrões internacionais, havendo a necessidade de se estabelecer um padrão de normalidade para a raça. A obesidade, que interfere na relação espacial da falange distal com o estojo córneo, indica que equinos Crioulos obesos estão mais propensos a desenvolver laminite. A ocorrência desses achados antes do aparecimento de sinais clínicos da doença e os indícios da influência da concentração plasmática de insulina aqui verificados encorajam novos estudos utilizandose o exame radiográfico do casco e a mensuração plasmática de insulina como possíveis métodos de diagnóstico precoce da doença em raças nacionais.

\section{AGRADECIMENTOS}

À Fundação de Amparo à Pesquisa do Estado de Minas Gerais (FAPEMIG), à Fundação de Amparo à Pesquisa do Estado do Rio Grande do Sul (FAPERGS), ao CNPq e à CAPES, pelo apoio financeiro.

\section{REFERÊNCIAS}

ASPLIN, K.E.; SILLENCE, M.N.; POLLITT, C.C. et al. Induction of laminitis by prolonged hyperinsulinaemia in clinically normal ponies. Vet. J., v.174, p.530-535, 2007.

BLACK, S.J.; LUNN, D.P.; YIN, C. et al. Leukocyte emigration in the early stages of laminitis. Vet. Immunol. Immunopathol., v.109, p.161-166, 2006.

CARTER, R.A.; GEOR, R.J.; BURTON STANIAR, W. et al. Apparent adiposity assessed by standardised scoring systems and morphometric measurements in horses and ponies. Vet. J., v.179, p.204-210, 2009.

DE LAAT, M.A.; SILLENCE, M.N.; McGOWAN, C.M. et al. Continuous intravenous infusion of glucose induces endogenous hyperinsulinaemia and lamellar histopathology in Standardbred horses. Vet. J., v.191, p.317-322, 2012.

COELHO, D.O. Nasce a raça Crioula. 2012. Disponível em: <http://www.racacrioula.com.br/ site/content/entidade/cavalo_crioulo.php>. Acessado em: 8 jun. 2012. 
FALEIROS, R.R.; NUOVO, G.J.; BELKNAP, J.K. Calprotectin in myeloid and epithelial cells of laminae from horses with black walnut extract-induced laminitis. J. Vet. Intern. Med., v.23, p.174-181, 2009.

FALEIROS, R.R.; NUOVO, G.J.; FLECHTNER, A.D. et al. Presence of mononuclear cells in normal and affected laminae from the black walnut extract model of laminitis. Equine Vet. J., v.43, p.45-53, 2011.

FRANK, N. Equine Metabolic Syndrome. Vet. Clin. Equine, v.27, p.73-92, 2011.

FRANK, N.; ELLIOT, S.; BRANDT, L. et al. Physical characteristics, blood hormone concentrations, and plasma lipid concentrations in obese horses with insulin resistance. J. Am. Vet. Med. Associa., v.228, p.1383-1390, 2006.

FRANK, N.; GEOR, R.J.; BAILEY, S.R. et al. Equine Metabolic Syndrome. J. Vet. Intern. Med., v.24, p.467-475, 2010.

HENNEKE, D.; POTTER, G.; KREIDER, J. et al. Relationship between condition score, physical measurements and body fat percentage in mares. Equine Vet. J., v.15, p.371-372, 1983.

HOLBROOK, T.C.; TIPTON, T.; McFARLANE, D. Neutrophil and cytokine dysregulation in hyperinsulinemic obese horses. Vet. Immunol. Immunopathol., v.145, p.283-289, 2012.

JOHNSON, P.J.; WIEDMEYER, C.E.; LACARRUBBA, A. et al. Laminitis and the equine metabolic syndrome Veterinary. Clin. N. Am.: Equine Pract., v.26, p.239-255, 2010.

KARIKOSKI, N.P.; HORN, I.; MCGOWAN, T.W. et al. The prevalence of endocrinopathic laminitis among horses presented for laminitis at a first-opinion/referral equine hospital. Domest. Anim. Endocrinol., v.41, p.111-117, 2011.
LIMA, I.R.; LIMA L.R.; MAGALHÃES, J.F. et al. Medidas radiográficas do dígito de éguas da raça Mangalarga Marchador com e sem sinais de obesidade. In: SIMPÓSIO INTERNACIONAL DO CAVALO ATLETA. 5., 2011, Belo Horizonte. Anais... Revista Veterinária e Zootecnia em Minas, Belo Horizonte, CRMVMG, 2011. v.109, p.75-76.

PASS, M.A.; POLLITT, S.; POLLITT, C.C. Decreased glucose metabolism causes separation of hoof lamellae in vitro: a trigger for laminitis? Equine Vet. J., v.26, p.133-138, 1998.

POLLITT C.C. Equine Laminitis Current Concepts. Publication No. 08/062, Project No. RIRDC UQ-118A. Queensland - Australia, 2008. p.55-60.

STASHAK, T.S. Claudicação em Equinos segundo Adams. São Paulo: Roca, 2006. p.95110.

TREIBER, K.H.; KRONFELD, D.S.; HESS, T.M. et al. Prelaminitic metabolic syndrome in genetically predisposed ponies involves compensated insulin resistance. J. Anim. Physiol. Anim. Nutr., v.89, p.430-431, 2005.

VISSER, M.B.; POLLITT, C.C. Lamellar leukocyte infiltration and involvement of IL-6 during oligofructose-induced equine laminitis development. Vet. Immunol. Immunopathol., v.144, p.120-128, 2011.

WALSH, D.M.; McGOWAN, C.M.; McGOWAN, T. et al. Correlation of plasma insulin concentration with laminitis score in a field study of Equine Cushing's Disease and Equine Metabolic Syndrome. J. Equine Vet. Sci., v.29, p.87-94, 2009. 